\title{
Effects of Interfacial Properties of a Surface Modified Surface Plasmon Resonance Chip on Protein Immobilization Performance
}

\author{
Juan Wu,* Yanbin JiANG,* Lishi WANG,* and Qiang ZENG*,**† \\ *School of Chemistry and Chemical Engineering, South China University of Technology, Guangzhou 510641, \\ People's Republic of China \\ **State Key Laboratory of Pulp and Paper Engineering, South China University of Technology, Guangzhou 510641, \\ People's Republic of China
}

\begin{abstract}
In order to confirm the correlation between interfacial properties of modified surface plasmon resonance (SPR) chips and their SPR responses to immobilized anti-IgG, SPR chips were modified by mercaptoundecanoic acid, poly(ethylene glycol) diacrylate (PEG), PEG-based copolymer and cyclodextrin coupled PEG using self-assembled or radical polymerization methods. The resulting interfacial properties such as film thickness and hydrophilicity were characterized by AFM, elliptic polarization scanners and contact angle meter. Immobilization of human IgG on the modified chips was achieved by EDC/NHS activation through an amide bond. The association between fixed IgG and free anti-IgG was reflected by the variation of SPR responses and the binding ability was evaluated by Langmuir isotherms. As observed, the adsorption between IgG and anti-IgG was affected by the interfacial properties of different modifiers, such that a chip with a thinner and more hydrophilic layer may result in a higher SPR response, producing a larger adsorption equilibrium constant for protein interaction.
\end{abstract}

Keywords Surface plasmon resonance array, surface modification, protein immobilization, nonspecific adsorption

(Received September 5, 2016; Accepted November 8, 2016; Published April 10, 2017)

\section{Introduction}

Surface plasmon resonance (SPR) is a physical optical phenomenon, ${ }^{1-4}$ which is sensitive to the dielectric constant of the medium. ${ }^{5}$ SPR has attracted interest for biosensing ${ }^{6-8}$ especially with respect to biomolecular interactions.9-11 By contrast with other reported analytical methods such as immunofluorescence, ${ }^{12,13}$ chromatographic analysis ${ }^{14,15}$ and mass spectrometry, ${ }^{16,17}$ which usually require expensive equipment and complicated operation, biosensors based on the SPR process offer many superior advantages, including but not limited to label-free and real-time detection, low sample consumption, less time-consuming process, high sensitivity and efficiency, and the strong capability of anti-electromagnetism. ${ }^{18,19}$ Therefore, SPR sensing has been widely used in applications in different fields e.g., medical diagnostics, biopharmaceutical, environmental monitoring and food security. ${ }^{20,21}$

However, SPR sensing has some deficiencies mainly due to strong nonspecific adsorption of the SPR chip surface, which limits this method in practical applications. ${ }^{22,23}$ To address this drawback, surface modified SPR chips can be considered. Common methods employed for surface modification of SPR chips are self-assembly and radical polymerization. ${ }^{24}$ The resulting layer on the SPR chip surface may minimize nonspecific adsorption of the chip and improve the sensitivity of SPR response by introducing the additional functionality of

$\dagger$ To whom correspondence should be addressed.

E-mail: ceqzeng@scut.edu.cn added terminal attaching groups for specific binding with target species. For example, Tothill et al. ${ }^{10}$ have reported the investigation of protein and DNA immobilization on Au surfaces by constructing different surface chemistries on SPR sensor chips. Results showed that the immobilization on the chip surface modified with self-assembled mercaptoundecanoic acid improved the sensitivity and produced the highest SPR response. On the other hand, Chilkoti et al. ${ }^{25}$ reported the modification of a gold SPR chip by a surface initiated polymerization of an oligo(ethylene glycol) methacrylate. The binding behavior of the resulting polymer brushes is mainly based on the polymer side chains and the polymer backbone otherwise exhibited excellent anti-nonspecific adsorption ability to proteins. Thus, the interfacial properties of modified SPR chips seem to exhibit significant impact on the protein sensing performance of SPR chips, reflected by the variation of SPR responses of chips. Interestingly, studies correlating to the relationship between interfacial properties of modifiers and chip performance are rare. ${ }^{26}$

In this contribution, we focus on finding possible correlations between surface properties of modified SPR chips and resulting SPR responses. The surface of SPR chips was initially modified with differently conventional layers of mercaptoundecanoic acid (MUA), poly(ethylene glycol) diacrylate (PEG), PEG-based copolymer and cyclodextrin coupled PEG by both selfassembled and radical polymerization methods. In particular, the surface properties of the PEG layer can be easily adjusted by the introduction of side chains or cyclodextrin molecules. Further, human IgG was attached on the chips through an amide bond by the process of EDC/NHS activation. ${ }^{27}$ The binding 
between the resulting chips and anti-IgG generated significant SPR responses and was figured by Langmuir isotherms. Simultaneously, the effects of different surface chemistry on the performance of SPR array were also investigated by exploring the relationship between interfacial properties of modifiers and chip performance in particular, for PEG-based modifiers. Therefore, deeper understanding of these effects is mandatory for further progress towards rational development of novel surface-modified SPR chips featuring anti-nonspecific adsorption ability and enhanced sensibility for biosensing applications.

\section{Experimental}

\section{Reagents and chemicals}

11-Mercaptoundecanoic acid (MUA, 95\%), thiol poly(ethylene glycol) $2 \mathrm{kDa}(95 \%)$, poly(ethylene glycol) diacrylate $2 \mathrm{kDa}$ (PEG, 95\%), and $\alpha$-cyclodextrin (CD, 98\%) were purchased from J\&K Chemical. Mercaptoundecyl bromoisobutyrate (95\%) and SPR chips were obtained from Plexera. Oligo(ethylene glycol) methacrylate (OEGMA), 2-hydroxyethyl methacrylate (HEMA), 1-(3-dimethylaminopropyl)-3-ethylcarbodiimide hydrochloride (EDC, 98.5\%), and $N$-hydroxysuccinimide (NHS, 98\%) were purchased from Aladdin. Glycine (analytical grade), human immunoglobulin $\mathrm{G}$ ( $\mathrm{IgG})$, FITC-labeled goat anti-human IgG were purchased from BoAoShen (Beijing, China). All materials were used as received without further purification.

\section{Surface modification of SPR chips}

Prior to fabrication of arrays, a gold-coated SPR chip was initially cleaned in a mixed solution containing $\mathrm{H}_{2} \mathrm{O}, \mathrm{H}_{2} \mathrm{O}_{2}$ $(30 \%)$ and the ammonia solution $(25 \%)(5: 1: 1)$ at $80^{\circ} \mathrm{C}$ for 10 min. After cooling down to room temperature, the chip was taken from the mixture and then rinsed with deionized water and ethanol three times. Finally, the chip was blown dry by pure $\mathrm{N}_{2}$ to completely remove the solvent residues.

For the PEG-based copolymer layer, the active poly(ethylene glycol) monolayers for further atom transfer radical polymerization (ATRP) were firstly self-assembled onto the surface of the as-prepared chips by immersing the chips in an ethanol solution containing $1 \mathrm{mM}$ of thiol poly(ethylene glycol) and mercaptoundecyl bromoisobutyrate for $15 \mathrm{~h}$. The monolayer was rinsed with deionized water and blown dry with $\mathrm{N}_{2}$ before use. For further polymerization, the monomer was prepared from $\mathrm{Cu}$ (II)-bpy (2,2'-bipyridine) complex with OEGMA and HEMA. Next, $12.5 \mathrm{mg}(0.8 \mathrm{mmol})$ of bpy ligand was added into $1 \mathrm{~mL}$ of $0.04 \mathrm{mM} \mathrm{CuCl}_{2}$ solution. When complexation was completed, the solution color changed from light blue to royal purple and then $2.62 \mathrm{~g}(5 \mathrm{mM})$ of OEGMA, $0.65 \mathrm{~g}(5 \mathrm{mM})$ of HEMA, $5 \mathrm{~mL}$ of pure water and $5 \mathrm{~mL}$ of methanol were mixed under stirring and $\mathrm{N}_{2}$ for $30 \mathrm{~min}$. After which time, the prepared chip was placed in this resulting solution for the surface polymerization. Under $\mathrm{N}_{2}, 1 \mathrm{~mL}$ of $0.04 \mathrm{mM}$ ascorbic acid was injected into the solution, resulting in the reduction of $\mathrm{Cu}$ (II) to $\mathrm{Cu}(\mathrm{I})$, which would trigger the surface polymerization. The polymerization on the chip surface was conducted at ambient temperature in a glove-box under $\mathrm{N}_{2}$ for $17 \mathrm{~h}$. The modified chip was collected after polymerization then cleaned with methanol and deionized water for further use. Moreover, the polymer on the chip surface was further functionalized with carboxyl terminals by immersing the chip in a DMF solution containing $10 \mathrm{mg} \mathrm{mL}^{-1}$ of 2,5-dioxotetrahydrofuran and $15 \mathrm{mg} \mathrm{mL}^{-1}$ of 4-dimethylamiopryidine (DMAP) at room temperature for $20 \mathrm{~h}$. The resulting chip was rinsed with DMF and ethanol and
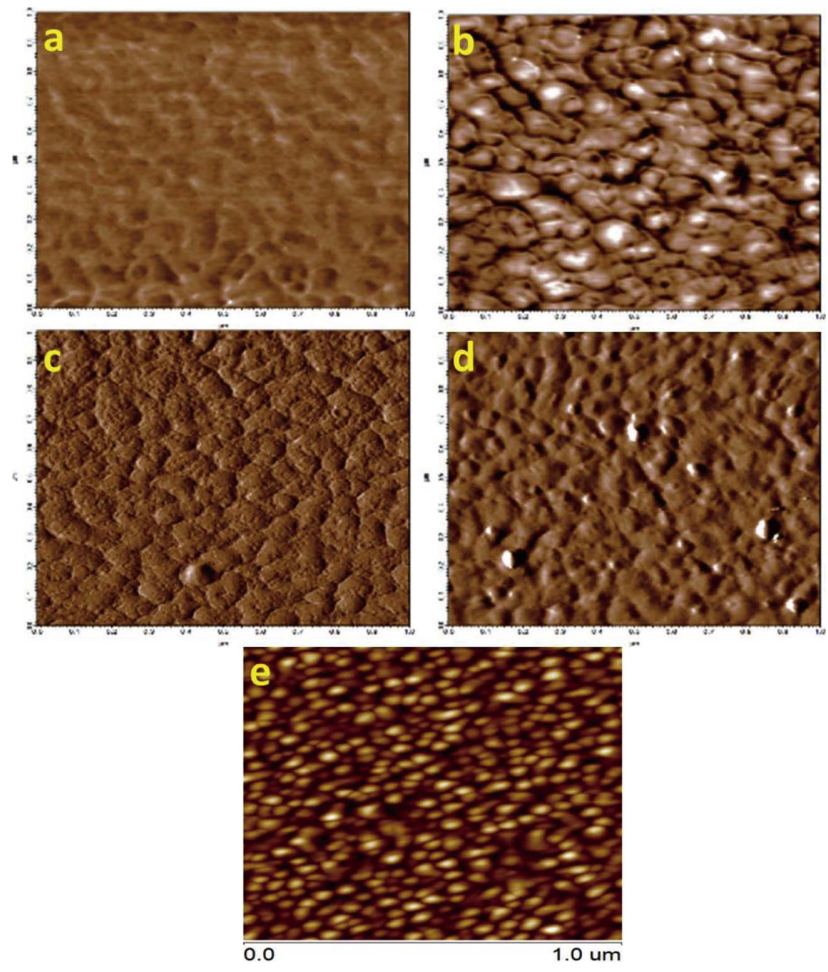

Fig. 1 Surface morphologies by AFM of SPR chips modified with (a) copolymer (OEGMA-co-HEMA), (b) MUA, (c) PEG, (d) CD-PEG and (e) unmodified.

blown dry with $\mathrm{N}_{2}$ before use.

The self-assembled MUA layer can be prepared by immersing the as-prepared chip in $1 \%$ of $10 \mathrm{mM}$ MUA solution for $14 \mathrm{~h}$, then rinsing with ethanol completely and blowing dry with $\mathrm{N}_{2}$ before use.

The preparation of the self-assembled PEG layer is similar to the MUA layer, the as-prepared chip is immersed in $1 \%$ of $10 \mathrm{mM}$ poly(ethylene glycol) diacrylate solution for $14 \mathrm{~h}$, then rinsed with ethanol and blown dry with $\mathrm{N}_{2}$ for further use.

For the CD coupled PEG (CD-PEG) layer, the cooperation between the self-assembled PEG layer and cyclodextrin molecules was achieved through a host-guest interaction by immersing the chip in a cyclodextrin aqueous solution for $4 \mathrm{~h}$. After which time, a EDC/NHSS (0.04/0.01 M) mixed solution was added to activate the $-\mathrm{COOH}$ terminal of PEG, which was further sealed by adding $200 \mu \mathrm{g} \mathrm{mL} \mathrm{mL}^{-1}$ of Z-Tyr-OH to prevent the escape of CD from the PEG layer. Finally, the layer was functionalized with carboxyl terminals in a DMF solution (10 mg mL $\mathrm{m}^{-1}$ of 2,5-dioxotetrahydrofuran and $15 \mathrm{mg} \mathrm{mL}^{-1}$ of DMAP) at room temperature for $20 \mathrm{~h}$. The resulting chip was ready for use after carefully rinsing with DMF and ethanol and blown dry with $\mathrm{N}_{2}$.

\section{Preparation of protein arrays}

A QArray mini printer from Genetix was utilized and the arrayer was equipped with a 384-well microplate, a pumped wash station, a dry station with air compressor and a robot for printing pins. The well-prepared chips were soaked in an aqueous solution containing $0.4 \mathrm{M}$ of EDC and $0.2 \mathrm{M}$ of NHS for activation in a shaker $(220 \mathrm{r} / \mathrm{min})$ for $30 \mathrm{~min}$. To immobilize human IgG on chips, the solution of NaAc-HAc and $20 \%$ of glycaral with a desired concentration of $\mathrm{IgG}$ was added to the microplate for printing and formed five sample spots at each 


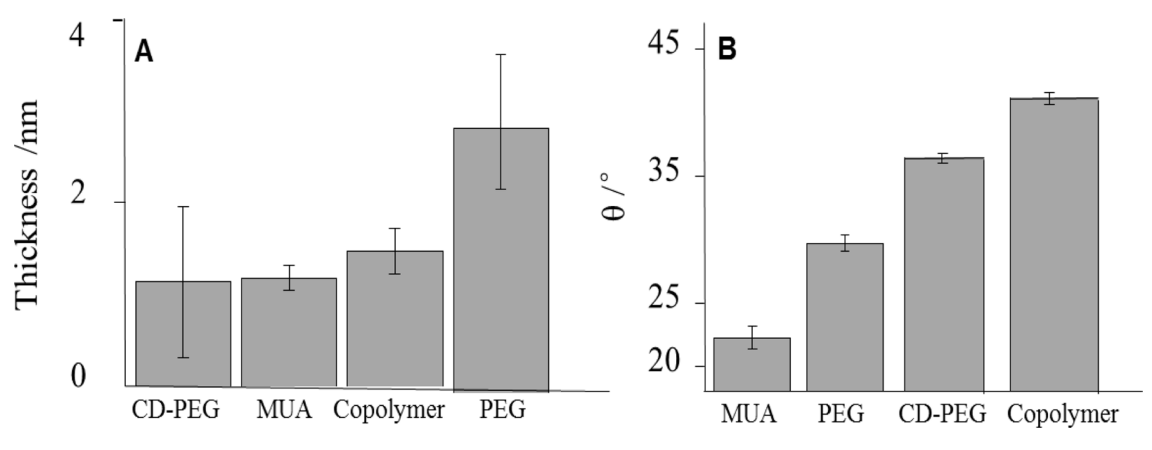

Fig. 2 Film thickness (A) and contact angle (B) of different modified SPR chips.

concentration. Before measurment, $1 \mathrm{~mL}$ of PBS-T buffer solution was pumped to sweep away unbound $\mathrm{IgG}$ from the chip surface, then $1000 \mu \mathrm{L}$ of ethanolamine $(1 \mathrm{M}, \mathrm{pH} 8.5)$ was employed to deactivate the chip and $2 \mathrm{~mL}$ of PBS-T buffer solution was finally used to make the system reach a steady state. The injection rate used in these procedures was $2 \mu \mathrm{L} \mathrm{s}^{-1}$.

\section{Apparatus}

The surface morphologies of different SPR chips were observed by AFM (EPMA1600, Japan). It was performed on a Bruker Multi Mode 8 tapping mode with the tip strength of at least 2.5 and vertical and horizontal variables between \pm 0.1 . The layer thickness on the surface of the SPR chip was monitored by a UV/SEL-NIR-FGMS spectroscopic elliptic polarization scanner $\left(1.5-5 \mathrm{eV}\right.$, angel at $\left.65^{\circ}\right)$ and the hydrophilic properties of the chips were characterized by a static contact angle method (OCA40 Micro, German). For SPR measurements, a high throughput and label free biomolecular interaction detection system based on Surface Plasmon Resonance imaging technology (PlexArray ${ }^{\circledR}$ HT, Plexera ${ }^{\circledR}$ LLC, USA) was adopted.

\section{Results and Discussion}

Surface characterization of modified SPR chips

Surface morphologies of SPR chips with different modifiers were gained by AFM images. In particular, the modifier of copolymer (OEGMA-co-HEMA) prepared by ATRP was displayed as a homogeneous polymer film (Fig. 1a), where the inlaid spots were proposed as OEGMA and HEMA side chains. MUA was immobilized on a SPR chip as a single molecule via a thiol functional group at its terminal and shown as protuberant portions at the surface of SPR chips (Fig. 1b). Self-assembled PEG film also grew on a SPR chip surface in the form of bulges (Fig. 1c), exhibiting a compact polymeric feature due to its larger molecular weight $(2 \mathrm{kDa})$. The density of these bulges of PEG film became less compact when CD coupled to PEG (Fig. 1d), suggesting changes of interfacial properties. By contrast, only regular and dense spots are observed on a bare SPR chip (Fig. 1e) and these spots can be assigned to a uniform gold surface. In summary, the modifiers are fully covered on the surface of SPR chips and the observed differences in surface morphologies confirm the modifications of SPR chips in different cases.

Prior to investigation of the association abilities of the surface modified SPR chips with a suitable protein, the interfacial properties of these SPR chips have been studied and may show significant impact on the degree of SPR response signals during measurements. The thickness of the thin layer modified on each SPR chip was measured by elliptic polarization scanner, following the order CD-PEG $<$ MUA $<$ copolymer $<$ PEG (Fig. 2A). A thinner modifier commonly requires less SPR energy, giving a much stronger resonant response signal. Therefore, the signals potentially generated on different modified chips may show an opposite order to the modifier thickness.

As the spotting sample of human $\operatorname{IgG}$ is usually prepared as an aqueous solution, the immobilization of IgG on SPR arrays is thus significantly affected by the hydrophilic properties of SPR chip surfaces. The spotting IgG sample on the surface of the SPR chip can be expanded more widely when the surface is hydrophilic, namely, resulting in a lower local concentration of immobilized IgG per unit area and further producing a weaker resonant response. In order to determine the hydrophilicity of each modified chip surface, the contact angle between the chip surface and the spotting aqueous solution is utilized for this purpose. For example, the SPR surfaces modified with MUA and PEG are more hydrophilic, showing a contact angle range from 22 to $30^{\circ}$ (Fig. 2B) due to the spreading of the sample solution. By contrast, copolymer (OEGMA-co-HEMA) and CD-PEG are less hydrophilic and it is in line with their larger contact angles. In particular, the former is due to the hydrophobic properties of OEGMA and HEMA chains and the latter is because of the hydrophobic cavity of cyclodextrin, which results in a drop of hydrophilic properties of the SPR chip modified by CD-PEG layer. The potential order of SPR response on different modified chips thus follows: MUA $<$ PEG $<$ CD-PEG $<$ copolymer (Fig. 2B).

\section{The optimum conditions of measurements}

The signal variation of the SPR spotting arrays is dependent upon the fixed concentration of IgG. For example, for the chip surface modified by copolymer (OEGMA-co-HEMA), a flat baseline was initially obtained by the flow of $1000 \mu \mathrm{L}$ PBS-T buffer solution (Fig. 3, A1). When a $25 \mathrm{nM}$ of anti-IgG solution was injected, SPR intensities gradually increased due to the association between the immobilized IgG and free anti-IgG (Fig. 3, A2). This process was influenced by the concentrations of fixed $\operatorname{IgG}$, demonstrating that the more proteins assembled on the chip surface resulted in the higher signal intensity, but it took a longer time to achieve association equilibrium with antiIgG. After intensities reached to maximum at $266 \mathrm{~s}$, the PBS-T buffer solution was introduced again, generating a series of flat steps (Fig. 3, A3). Then, $800 \mu \mathrm{L}$ of $100 \mathrm{mM}$ glycine (pH 2.0) was pumped to the chips to remove the associated anti-IgG, showing a significant decrease of intensities (Fig. 3, A4). Finally, the flat baselines were fully recovered with the 

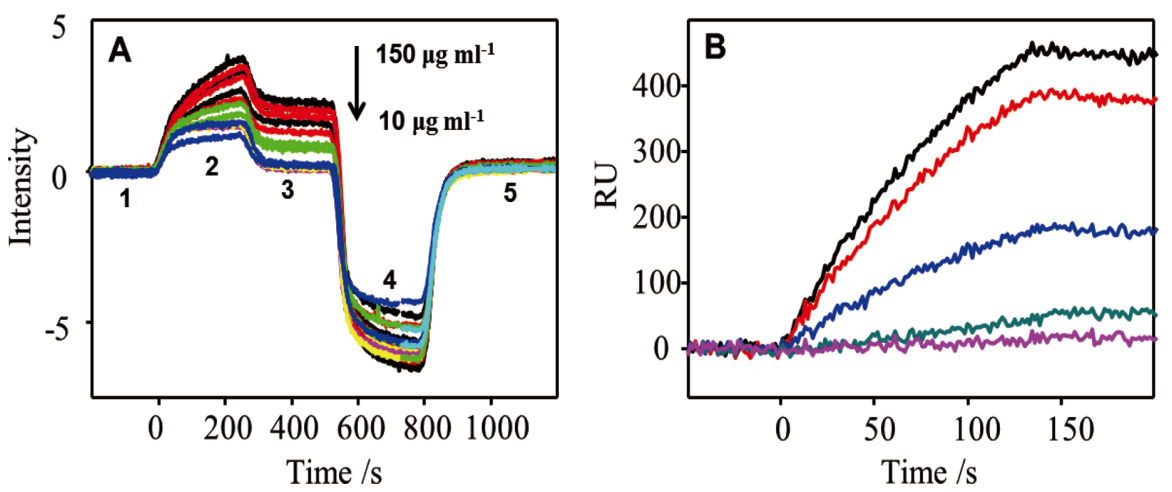

Fig. 3 (A) The regeneration process of the SPR spotting arrays with surface modification of copolymer (OEGMA-co-HEMA) where immobilized IgG on the chip surface varies from 10 to $150 \mu \mathrm{g} \mathrm{mL}^{-1}$. (B) SPR responses to $25 \mathrm{nM}$ anti-IgG where the chip fixed with $100,75,50,25$ and $12.5 \mu \mathrm{g} \mathrm{mL}^{-1}$ of IgG (from top to bottom), respectively.
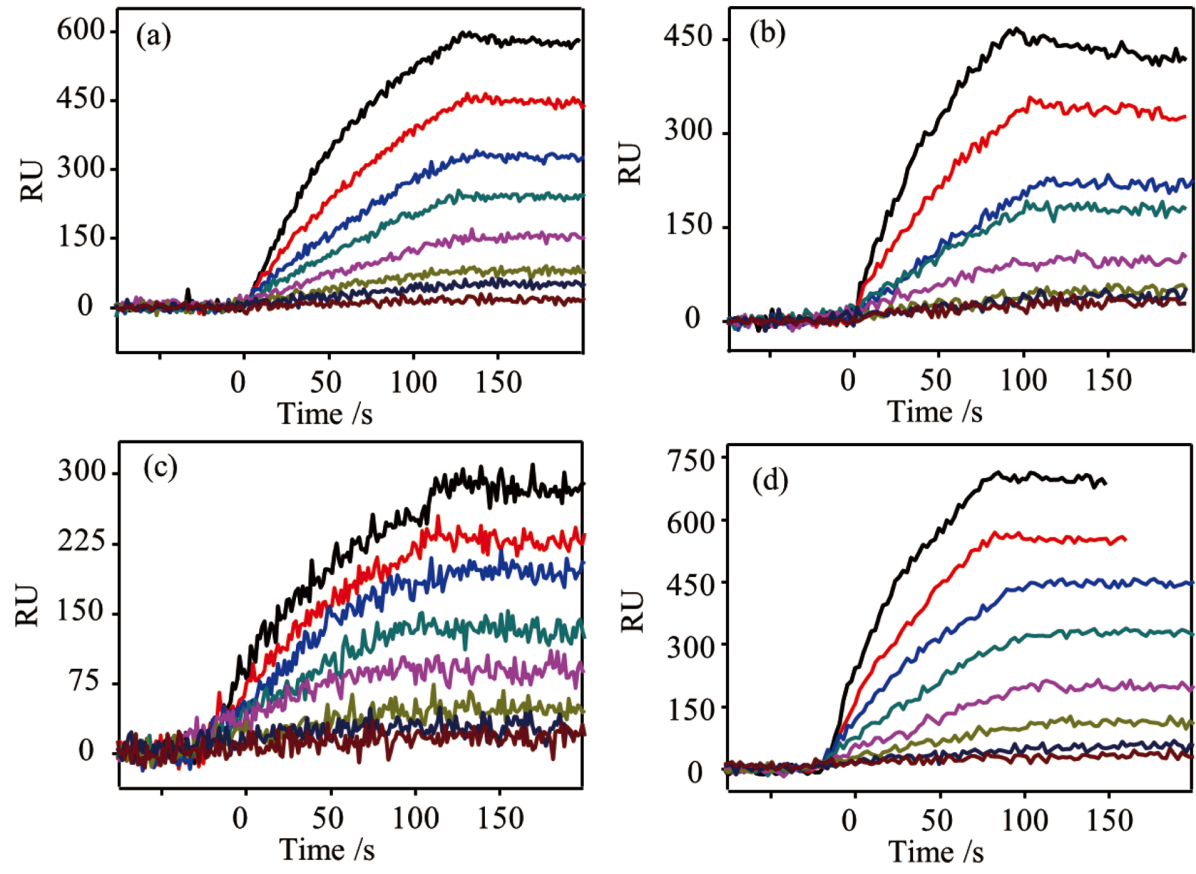

Fig. 4 Responses of fixed human $\operatorname{IgG}\left(100 \mu \mathrm{g} \mathrm{mL}^{-1}\right)$ to different concentrations of anti-IgG (from top to bottom: 50, 25, 15, 10, 5, 2.5, 1 and $0.5 \mathrm{nM}$ ) on the SPR spotting arrays with surface modification of (a) copolymer (OEGMA-co-HEMA), (b) MUA, (c) PEG (d) CD-PEG.

introduction of the PBS-T buffer solution again (Fig. 3, A5) This observation was also noted on the chips with other modifiers and suggests that the bound anti-IgG was completely removed from the chips and the chips represent excellent reproducibility.

The specific adsorption intensity can be converted with the unit of RU, which is obtained from the difference between buffer baselines. In order to achieve reasonable measuring time for the association between the immobilized IgG and free anti$\mathrm{IgG}$, the fixed $\mathrm{IgG}$ concentration of $100 \mu \mathrm{g} \mathrm{mL}^{-1}$ was chosen and employed, where association equilibrium was obtained in less than $200 \mathrm{~s}$ by producing relatively high sensing response (Fig. 3B). These conditions were also applied to other chips used in this work and utilized in further experiments.
Association abilities to anti-IgG

The association ability between $\mathrm{IgG}$ and anti-IgG on each modified SPR chip was investigated by the antigen-antibody binding curve (Fig. 4). As revealed by Fig. 4, the SPR chips modified with CD-PEG and copolymer (OEGMA-co-HEMA) can easily distinguish different concentrations of anti-IgG when stabilized with a certain amount of human $\operatorname{IgH}$ and produce relatively higher response signals. By contrast, the chips with the modification layers such as MUA and PEG represent lower SPR maximum response values and poorer signal resolution, in particular, with low concentrations of anti-IgG.

When the adsorption and desorption reach dynamic equilibrium, the number of adsorbed antibody molecules on the chip surface was relatively stable. The dynamic adsorption and desorption equilibrium process can be regarded as a single 

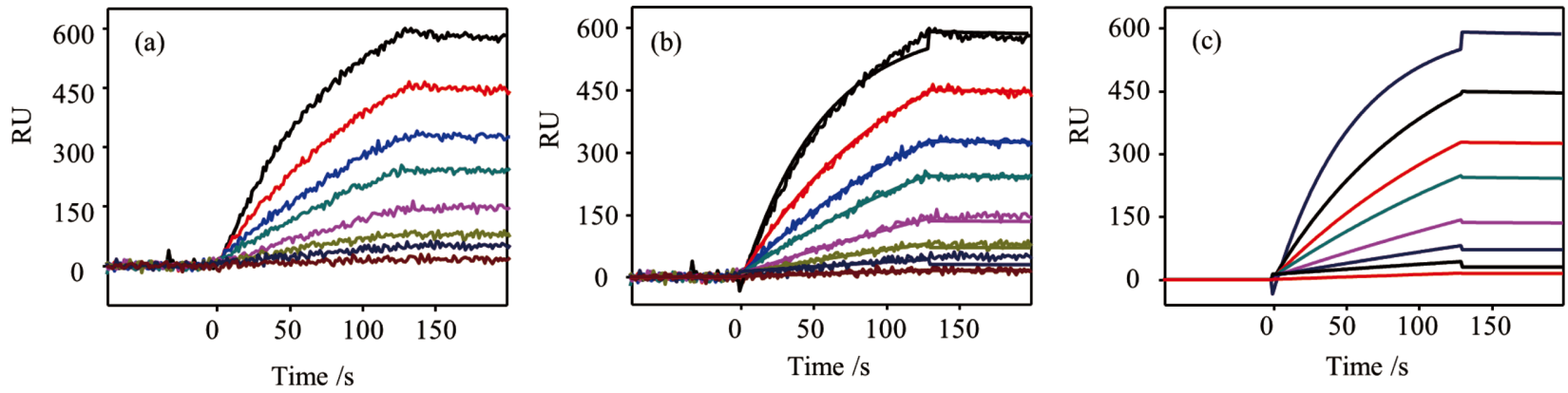

Fig. 5 The assumed responses between fixed human $\operatorname{IgG}\left(100 \mu \mathrm{g} \mathrm{mL}^{-1}\right)$ and different concentrations of anti-IgG (from top to bottom: 50, 25, 15, 10, 5, 2.5, 1 and $0.5 \mathrm{nM}$ ) on the chip with modification of copolymer (OEGMA-co-HEMA) by BIAevaluation in a Langmuir binding mode, (a) original responses, (b) best fit and (c) fitted responses.

Table 1 Kinetic constants $\left(K_{\mathrm{a}}, K_{\mathrm{d}}, K_{\mathrm{D}}\right)$ of different chips

\begin{tabular}{lccc}
\hline \multicolumn{1}{c}{ Modifier } & $K_{\mathrm{a}} / \mathrm{M} \mathrm{s}^{-1}$ & $K_{\mathrm{d}} / \mathrm{s}^{-1}$ & $K_{\mathrm{D}} / \mathrm{M}^{-1}$ \\
\hline Copolymer & $3.66 \times 10^{5}$ & $2.91 \times 10^{-6}$ & $1.26 \times 10^{11}$ \\
MUA & $6.57 \times 10^{5}$ & $4.52 \times 10^{-4}$ & $1.45 \times 10^{9}$ \\
PEG & $5.32 \times 10^{5}$ & $4.30 \times 10^{-4}$ & $1.24 \times 10^{9}$ \\
CD-PEG & $9.55 \times 10^{5}$ & $2.01 \times 10^{-6}$ & $4.76 \times 10^{11}$ \\
\hline
\end{tabular}

molecule layer adsorption. Assuming that the surface adsorption force was uniform and the interaction between adsorbed molecules was very small, a Langmuir isotherm can be applied to describe the adsorption equilibrium constant to better understand the binding ability between immobilized human IgG and free anti-body. By BIAevaluation (sampled by the chip with modification of copolymer (OEGMA-co-HEMA) in Fig. 5), the Langmuir (1:1) binding model is capable of accurately reproducing the association and disassociation progresses using two kinetic parameters, i.e., $k_{\mathrm{a}}$ and $k_{\mathrm{d}}{ }^{28}$ According to Eq. (1) a linear correlation between the SPR signal and time is observed, giving a slope of $k_{\mathrm{obs}}$, where $k_{\mathrm{obs}}=k_{\mathrm{a}} f_{0}+k_{\mathrm{d}}$. At different concentrations, $k_{\text {obs }}$ linearly changes with the variation of concentration $f_{0}$ and the related slope and intercept are described as $k_{\mathrm{a}}$ and $k_{\mathrm{d}}$, respectively. Furthermore, the adsorption equilibrium constant $\left(K_{\mathrm{D}}\right)$ of each modified chip is expressed as Eq. (2) and summarized in Table 1. The observed $K_{\mathrm{D}}$ values between IgG and anti-IgG for the chips with different modifiers closely correlate with their SPR maximum response values, presenting their specific sensitivity. In particular, human $\mathrm{IgG}$ on the chips decorated with copolymer (OEGMA-coHEMA) and CD-PEG shows much stronger binding ability to free anti-body. Significantly, these apparent $K_{\mathrm{D}}$ values are more than two orders of magnitude larger than those for the chips with MUA and PEG modifiers. This result suggests that the type of the modifiers may play an important role in the contribution of binding process between $\mathrm{IgG}$ and anti-IgG.

$$
\begin{aligned}
\frac{\mathrm{d} R}{\mathrm{~d} T} & =k_{\mathrm{a}} f_{0} R_{\text {eq }}-\left(k_{\mathrm{a}} f_{0}+R_{\mathrm{d}}\right) R_{\mathrm{t}} \\
K_{\mathrm{D}} & =\frac{k_{\mathrm{a}}}{k_{\mathrm{b}}}
\end{aligned}
$$

\section{Effects of interfacial properties on binding}

$K_{\mathrm{D}}$ represents the binding ability between fixed $\mathrm{IgG}$ and free anti-IgG and directly reflects the SPR sensitivity of the chips with different modifiers. The trend of $K_{\mathrm{D}}$ variation of different chips is somehow associated with thickness and hydrophilic properties of different modifiers on the chips. As mentioned above, the chip with a thinner modifier would produce a stronger SPR signal. The chip with PEG polymer has the thickest modified layer, thus resulting in a lower $K_{\mathrm{D}}$ value. The thickness of CD-PEG is significantly reduced due to the introduction of CD molecules to the PEG layer and it would be responsible for the increased $K_{\mathrm{D}}$. The thickness of MUA and copolymer (OEGMA-co-HEMA) modifiers is a little bit larger but still comparable to that of CD-PEG. However, $K_{\mathrm{D}}$ value of the chip with MUA layer becomes more than two orders of magnitude lower than that obtained from the chips with CD-PEG. Therefore, the effect of hydrophilic properties of SPR chip surfaces should be accounted for where a hydrophilic SPR chip surface would lead to a weaker resonant response.

Copolymer (OEGMA-co-HEMA) and CD-PEG modified chip surfaces are less hydrophilic. Combined with their layer thickness, these properties are in good agreement with the observation that IgG fixed on the chips with copolymer (OEGMA-co-HEMA) and CD-PEG modifiers has larger $K_{\mathrm{D}}$ to free anti-IgG. As the MUA layer, it has reasonable thickness and should thus exhibit relatively strong response when fixed IgG associated with anti-IgG. But the pronounced hydrophilic property of MUA would cause a decrease of fixed IgG local concentration on MUA and further minimize the active binding sites to free anti-IgG. As a consequence, it shows poor binding ability to anti-IgG and results in a lower $K_{\mathrm{D}}$ value. Therefore, a correlation between surface properties of modified SPR chips and their SPR responses to anti-IgG can be concluded; a chip modified with a thinner and more hydrophilic layer allows for a higher SPR response, giving a larger adsorption equilibrium constant for surface protein interaction.

\section{Nonspecific adsorption between modifiers and anti-IgG}

It is very common that SPR arrays exhibit some degree of nonspecific adsorption, in particular, on bare gold chips. Nonspecific adsorption ability thus becomes a key factor to evaluate the performance of the SPR chip. In order to achieve this objective, the nonspecific adsorption performance of each modified chip was directly tested with anti-IgG without immobilization of IgG (Fig. 6). It should be proposed to present a zero nonspecific adsorption response for anti-IgG since there is no specific association between fixed modifiers and free anti-IgG. Among these modifiers, the MUA layer shows most significant nonspecific adsorption to anti-IgG with the highest response signal probably because of the short chain length of 


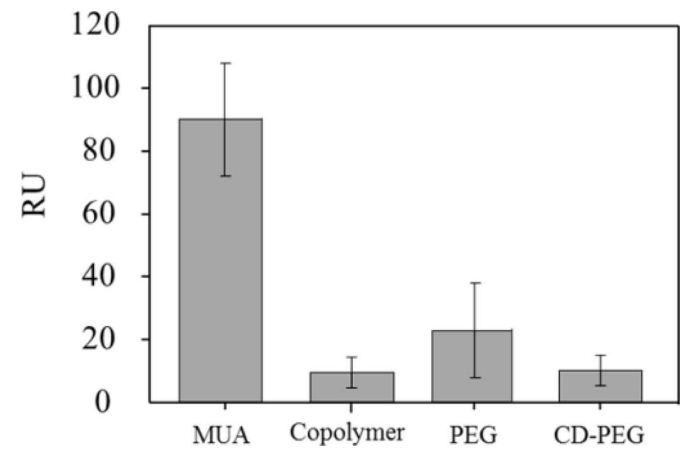

Fig. 6 Non-specific adsorption of different modifers to free anti-IgG.

the self-assembled MUA layer that allows the adsorption of anti-IgG to the gold chips. This feature also can be described as the origin for the poor resolution of SPR responses addressed above. By contrast with PEG layer, copolymer (OEGMA-coHEMA) and CD-PEG layers are not sensitive to anti-IgG and this can be assigned to their more compact structures due to the introduction of OEGMA and HEMA side chains and cyclodextrin macromolecules. These results clearly indicate the structure effect of different modifiers on nonspecific adsorption and it would be a factor that influences the SPR response of the association between fixed IgG and free anti-IgG in particular, for the MUA layer.

\section{Conclusions}

SPR chips were surface modified by copolymer (OEGMA-coHEMA), MUA, PEG and CD-PEG through self-assembled and radical polymerization methods, respectively. The different modifiers exhibited distinguished interfacial properties with orders of thickness: CD-PEG < MUA < copolymer < PEG and hydrophilicity: MUA < PEG < CD-PEG < copolymer. After further modification of human $\operatorname{IgG}$ through an amide bond by the EDC/NHS activation, the SPR chips showed specific adsorption to free anti-IgG proteins under optimized conditions and the association ability of each chip was evaluated by a Langmuir isotherm based on the variation of SPR responses. The results clearly demonstrated the fact that the association between $\operatorname{IgG}$ and anti-IgG can be a manner of chip surface with a modifier. A thinner and more hydrophilic modifier on a chip surface would result in higher SPR responses with a larger adsorption equilibrium constant for protein interaction, thus giving an order PEG $<$ MUA $<$ copolymer $<$ CD-PEG.

\section{Acknowledgements}

This research was supported by the National Natural Science Foundation of China (No. 21276091) and Ph.D. Programmes Foundation of the Ministry of Education of China (No. 20120172110010). We also acknowledge the State Key Laboratory of Pulp and Paper Engineering (201623) and the Fundamental Research Funds for the Central Universities (No. 2015ZM050). The authors appreciate Dr. Jinsong Zhu and Dr. Jian-An Liu of Plexera ${ }^{\circledR}$ LLC for providing the ultra-high throughput SPR imaging instrument and their thoughtful technical support.

\section{References}

1. D. M. Jones and W. T. S. Huck, Adv. Mater, 2001, 13, 1256.

2. W. Huang, J-B. Kim, M. L. Bruening, and G. L. Baker, Macromolecules, 2002, 35, 1175.

3. K. Matyjaszewski, D. A. Shipp, J-L. Wang, T. Grimaud, and T. E. Patten, Macromolecules, 1998, 31, 6836.

4. H. Yu, X. Shan, S. Wang, H. Chen, and N. Tao, Anal. Chem., 2014, 86, 8992.

5. M. Couture, S. S. Zhao, and J-F. Masson, Phys. Chem. Chem. Phys., 2013, 15, 11190.

6. P. N. Abadian and E. D. Goluch, Anal. Methods, 2015, 7, 115.

7. X. Wang, J. Xu, C. Liu, and Y. Chen, RSC Adv., 2016, 6, 21900.

8. P. Pereira, C. Cruz, J. A. Queiroz, A. Figueiras, and F. Sousa, Anal. Methods, 2016, 8, 205.

9. L. He, M. D. Musick, S. R. Nicewarner, F. G. Salinas, S. J. Benkovic, M. J. Natan, and C. D. Keating, J. Am. Chem. Soc., 2000, 122, 9071.

10. Z. Altintas, Y. Uludag, Y. Gurbuz, and I. Tothill, Anal. Chim. Acta, 2012, 712, 138.

11. K. P. M. Geuijen, L. A. Halim, H. Schellekens, R. B. Schasfoort, R. H. Wijffels, and M. H. Eppink, Anal. Chem., 2015, 87, 8115 .

12. J. J. Gooding, F. Mearns, W. Yang, and J. Liu, Electroanaysis, 2003, 15,81 .

13. J. S. Wang and K. Matyjaszewski, J. Am. Chem. Soc., 1995, $117,5614$.

14. M. Kato, M. Kamigaito, M. Sawamoto, and T. Higashimura, Macromolecules, 1995, 28, 1721.

15. W. Tang, N. V. Tsarevsky, and K. Matyjaszewski, J. Am. Chem. Soc., 2006, 128, 1598.

16. X. Huang and M. J. Wirth, Anal. Chem., 1997, 69, 4577.

17. M. Ejaz, S. Yamamoto, K. Ohno, Y. Tsujii, and T. Fukuda, Macromolecules, 1998, 31, 5934.

18. K. Matyjaszewski, P. J. Miller, N. Shukla, B. Immaraporn, A. Gelman, B. B. Luokala, T. M. Siclovan, G. Kickelbick, T. Vallant, H. Hoffmann, and T. Pakula, Macromolecules, 1999, 32, 8716.

19. X. Wang, J. Yu, A. Sreekumar, S. Varambally, R. Shen, D. Giacherio, R. Mehra, J. E. Montie, K. J. Pienta, M. G. Sanda, P. W. Kantoff, M. A. Rubin, J. R. Wei, D. Ghosh, and A. M. Chinnaiyan, New Engl. J. Med., 2005, 353, 1224.

20. W. Jakubowski and K. Matyjaszewski, Angew. Chem. Int. Ed., 2006, 45, 4482.

21. E. Wischerhoff, K. Uhlig, A. Lankenau, H. G. Börner, and A. Laschewsky, Angew. Chem. Int. Ed., 2008, 47, 5666.

22. P. Jain, J. Dai, G. L. Baker, and M. L. Bruening, Macromolecules, 2008, 41, 8413.

23. K. M. Mayer and J. H. Hafner, Chem. Rev., 2011, 111, 3828.

24. R. Barbey, L. Lavanant, D. Paripovic, N. Schüwer, C. Sugnaux, S. Tugulu, and H-A. Klok, Chem. Rev., 2009, 109, 5437.

25. H. Ma, J. Hyun, P. Stiller, and A. Chilkoti, Adv. Mater, 2004, 16, 338.

26. L. Liu, B. Ercan, L. Sun, K. S. Ziemer, and T. J. Webster, ACS Biomater. Sci. Eng., 2016, 2, 122.

27. D. A. Proshlyakov, T. Ogura, K. Shinzawa-Itoh, S. Yoshikawa, and T. Kitagawa, Biochemistry, 1996, 35, 76.

28. W. Beckmann, Opt. Eng., 2003, 3, 113. 